\title{
BOOLEAN POWERS: DIRECT DECOMPOSITION AND ISOMORPHISM TYPES
}

\author{
BY
}

\author{
KENNETH HICKIN AND J. M. PLOTKIN
}

\begin{abstract}
We determine properties of Boolean powers of groups and other algebraic structures, and we generalize Jónsson's theorem on Boolean powers of centerless, directly indecomposable groups. We show that every nonabelian, finitely generated group has $2^{\kappa_{0}}$ nonisomorphic countable Boolean, and hence subcartesian, powers. We show that nonabelian groups $G$ such that either (i) $G$ is not the central product of two nonabelian groups or (ii) every pair of nontrivial normal subgroups of $G$ intersect nontrivially yield nonisomorphic Boolean powers with respect to nonisomorphic Boolean algebras.
\end{abstract}

Summary. We determine properties of Boolean powers of groups and other algebraic structures in the spirit of Jónsson [4] and Lawrence [6]. In \$1 our main theorem concerns direct decompositions of Boolean powers and generalizes in a functorial setting Jónsson's well-known theorem on Boolean powers of centerless indecomposable groups. In $\$ 2$ we show that every nonabelian, finitely generated group has $2^{\kappa_{0}}$ nonisomorphic countable Boolean, and hence subcartesian, powers. (For regular $\kappa>\boldsymbol{\kappa}_{0}$ the existence of $2^{\kappa}$ mutually nonembeddable subcartesian powers of power $\kappa$ of every nonabelian group of power $<\kappa$ has been proved by Shelah using unstable theories.) We also show the following groups $G$ to be Boolean separating in Lawrence's sense that Boolean powers over $G$ separate the isomorphism types of all Boolean algebras: $G$ is nonabelian but $G$ is not the central product of two nonabelian groups; $G$ is nonabelian and every pair of nontrivial normal subgroups of $G$ intersect nontrivially (such groups are finitely subdirectly irreducible in Jónsson's terminology).

1. Let $B$ be a Boolean algebra and let $X$ be its Stone space. $B$ can be identified with the Boolean algebra of clopen subsets of the compact, 0-dimensional, Hausdorff space $X$. Let $\mathfrak{A}$ be an algebraic structure. $C(X, \mathfrak{A})$ is the set of all continuous functions from $X$ into $\mathfrak{A}$ where $\mathfrak{A}$ has the discrete topology. $C(X, \mathfrak{A})<\mathfrak{A}^{X}$ is an algebraic structure (under pointwise operations) of the same similarity type as $\mathfrak{A}$ and is called the bounded Boolean power of $\mathfrak{A}$ with respect to $B . C(X, \mathfrak{A})$ is thus a certain subcartesian power of $\mathfrak{A}$ whose members have finite range in $\mathfrak{A}$ corresponding to a finite partition of $1 \in B$ (finite from the compactness of $X$ ).

RemarK. To define the Boolean power any set representation $b \rightarrow X_{b}<X$ of $B$ as subsets of a set $X$ can be used. But we have a special use for the ultrafilters of $B$ (i.e. the elements of the Stone space $X$ ), first noticed by Jónsson [4], to project by

Received by the editors April 18, 1980.

1980 Mathematics Subject Classification. Primary 08A99; Secondary 20F99, 20E10, 06E99. 
point-evaluation direct factors of Boolean powers of $\mathfrak{A}$ back to direct factors of $\mathfrak{A}$. Elements of the Stone space can be selected via discontinuity of this direct factor point evaluation map. A single "discontinuous" direct factor of $C(X, \mathfrak{A})$ yields by projection a local system of proper direct factors of $\mathfrak{A}$. So, using the Stone space is very natural. (See Theorem 1 for details.)

Of particular interest to us are cases where $\mathfrak{A}$ is a structure possessing direct factors which themselves can form a Boolean algebra. Such structures are discussed by Fell and Tarski [3]. We will restrict our proofs to the case where $\mathfrak{A}=G$ is a group while keeping in mind that similar results hold for rings and many other systems.

Let $f \in C(X, \mathfrak{A})$. Since $f$ is continuous, $X$ is compact, and $\mathfrak{A}$ is discrete, there are distinct $a_{i} \in \mathfrak{A}$ and pairwise disjoint clopen subsets $U_{i}$ of $X(1<i<n)$ such that $X=\cup\left\{U_{i} \mid 1 \leqslant i \leqslant n\right\}$ and $U_{i}=f^{-1}\left(a_{i}\right)$. Thus $f$ has constancy sets corresponding to a finite partition of $1 \in B$.

Terminology. $U, V, U_{i}, V_{i}, \ldots$ always denote clopen subsets of $X . \bar{U}$ is the complement of $U$. $G$ always denotes a group and all groups are written in additive notation. $\Delta(G)$ and $\Delta(X, G)$ denote the sets of subgroups of $G$ and $C(X, G)$ which are direct factors of $G$ and $C(X, G)$ respectively.

For $U \subseteq X$ and $g \in G$, let $f(U, g): X \rightarrow G$ be

$$
f(U, g)(x)= \begin{cases}g, & x \in U, \\ 0, & x \notin U .\end{cases}
$$

Clearly $f(U, g) \in C(X, G)$. From the previous paragraph each $h \in C(X, G)$ has a unique representation as $f\left(U_{1}, g_{1}\right)+\cdots+f\left(U_{n}, g_{n}\right)$ where $\left\{U_{i} \mid 1<i<n\right\}$ partitions $X$ and $g_{1}, \ldots, g_{n}$ are distinct elements of $G . C(X, G)$ is generated by $\{f(U, g) \mid U \subseteq X, g \in G\}$. For $D<G, U \subseteq X$ we define $f(U, D)=\{f(U, g) \mid g \in$ $D\} . f(U, D)$ is a subgroup of $C(X, G)$. For $D \in \Delta(G)$ we also define $\delta(U, D)$ to be the subgroup of $C(X, G)$ generated by the groups $\{f(V, D) \mid V \subseteq U\}$.

LeMma. $\delta(U, D) \in \Delta(X, G)$.

Proof. Let $G=D \oplus D_{0}$. From the unique representation for elements of $C(X, G)$ it is easily seen that

$$
C(X, G)=\delta(U, D) \oplus \delta\left(U, D_{0}\right) \oplus \delta(\bar{U}, G)
$$

For our main theorem we need to assume that $\Delta(G)$ and $\Delta(X, G)$ are Boolean algebras under set intersection and algebraic join. The following simple theorem is contained in the results of Fell and Tarski [3]. Let $\zeta G$ be the center of the group $G$.

THEOREM 0. The following conditions on $G$ are equivalent:

(a) $\Delta(G)$ is a Boolean algebra under set intersection and algebraic join;

(b) $\Delta(G)$ has unique complementation;

(c) For all $D \in \Delta(G)$ and all $\varphi \in \operatorname{Hom}(G, \zeta G)$ we have $\varphi(D)<D$.

Under these conditions we say $\Delta(G)$ is Boolean. 
A similar theorem holds for a more general class of algebraic structures in which centers and internal direct decompositions can be defined relative to a distinguished binary operation. The theory includes, for example, the direct decomposition of a ring as the sum of two-sided ideals. In this situation the "center" of a ring is its two-sided annihilator. We refer the reader to [3] and [5].

We need also to consider when $\Delta(X, G)=\Delta(C(X, G))$ is Boolean.

Lemma 1. (a) If $\operatorname{Hom}(G, \zeta G)=0$ then $\operatorname{Hom}(C(X, G), \zeta C(X, G))=0$ and $\Delta(X, G)$ is Boolean.

(b) If $B \neq\{0,1\}$ then $\operatorname{Hom}(G, \zeta G)=0$ if and only if $\Delta(X, G)$ is Boolean.

Proof. (a) Clearly $\zeta C(X, G)=C(X, \zeta G)$. Let $\Phi \in \operatorname{Hom}(C(X, G), \zeta C(X, G))$. Suppose that, for some $f(U, g), \Phi(f(U, g)) \neq 0$. Then, for some $x \in X$, $\pi_{x} \circ \Phi(f(U, g))$ is a nonzero element of $\zeta G$ where $\pi_{x}$ is projection on the $x$ th coordinate. Let $\varphi$ embed $G$ in $C(X, G)$ via $h \mapsto f(U, h)$. Then $\pi_{x} \circ \Phi \circ \varphi$ is a nontrivial element of $\operatorname{Hom}(G, \zeta G)$. Thus $\Phi \equiv 0$.

(b) Assume $B \neq\{0,1\}$ and $0 \neq \varphi \in \operatorname{Hom}(G, \zeta G)$. Since $B \neq\{0,1\}$, we can choose $U$ such that $\varnothing \neq U \neq X$. Choose $x \in U$. Now $C(X, G)=\delta(U, G) \oplus$ $\delta(\bar{U}, G)$. Define $\Phi \in \operatorname{Hom}(C(X, G), \zeta C(X, G))$ as follows: $\Phi(\delta(\bar{U}, G))=0$ and for all $h \in \delta(U, G), \Phi(h)=f\left(\bar{U}, \varphi \circ \pi_{x}(h)\right)$. Clearly $\Phi(\delta(U, G)) \nless \delta(U, G)$ since $\varphi$ is nontrivial. So by part (c) of Theorem $0, \Delta(X, G)$ is not Boolean.

For $A \in \Delta(G)$ (resp. $\Delta(X, G)), \pi_{A}$ is the projection of $G$ (resp. $C(X, G)$ ) onto the direct factor $A . \pi_{A}$ is unique provided $\Delta(G)$ (resp. $\Delta(X, G)$ ) is Boolean.

Projection Lemma. Assume $\operatorname{Hom}(G, \zeta G)=0$. Suppose $\mathscr{D} \in \Delta(X, G), h \in \mathscr{D}$, and $V \subseteq h^{-1}(g)$ for some $g \in G$. Then $f(V, g) \in \mathscr{D}$.

Proof. Let $C(X, G)=\mathscr{D} \oplus \mathscr{D}_{0}$. We have $\delta(V, G) \in \Delta(X, G)$. Consider the homomorphism $\pi=\pi_{\mathscr{D}_{0}} \circ \pi_{\delta(V, G)} \circ \pi_{\mathscr{D}}$. Since $\mathscr{D}$ and $\mathscr{D}_{0}$ are complementary and projections increase centralizers, $\pi: C(X, G) \rightarrow \zeta C(X, G)$. So, by our assumption, $\pi$ must be trivial. Now $\pi_{\mathscr{D}}(h)=h$ and $\pi_{\delta(V, G)}(h)=f(V, g)$. Thus $\pi(h)=\pi_{\mathscr{D}_{0}}(f(V, g))$ $=0$ proving that $f(V, g) \in \mathscr{Q}$.

For $x \in X$ and $\mathscr{D} \in \Delta(X, G)$ let $G(x, \mathscr{D})=\{f(x) \mid f \in \mathscr{D}\}, G(x, \mathscr{D})=\pi_{x}(\mathscr{D})$ $\leqslant G$.

Lemma. Assume $\operatorname{Hom}(G, \zeta G)=0$ and $C(X, G)=\mathscr{D} \oplus \mathscr{D}_{0}$. Then $G=G(x, \mathscr{D})$ $\bigoplus G\left(x, \mathscr{D}_{0}\right)$.

Proof. By considering constant functions one can see $G=G(x, \mathscr{D})+$ $G\left(x, \mathscr{D}_{0}\right)$. That $G(x, \mathscr{D}) \cap G\left(x, \mathscr{D}_{0}\right)=\{0\}$ follows from $\mathscr{D} \cap \mathscr{D}_{0}=\{0\}$ and the projection lemma.

With $\operatorname{Hom}(G, \zeta G)=0$ and $\mathscr{D} \in \Delta(X, G)$ fixed, the function $x \mapsto G(x, \mathscr{D})$ maps $X$ into $\Delta(G)$. We denote this mapping by $G(, \mathscr{D})$. After endowing $\Delta(G)$ with the discrete topology, it makes sense to ask about the continuity of $G(, \mathscr{D})$.

Definition. $G$ has local direct factors if every finite subset of $G$ is contained in a proper direct factor of $G$. 
Theorem 1. Suppose $\operatorname{Hom}(G, \zeta G)=0$ and $\mathscr{D} \in \Delta(X, G)$. If $G(, \mathscr{D})$ is not continuous, then $G$ has local direct factors.

Proof. $G(, \mathscr{D})$ not continuous means there is an $A \in \Delta(G)$ such that $S=\{p \in$ $X \mid G(p, \mathscr{D})=A\}$ is not open. Hence there is a $p_{0} \in S$ such that for each $U$ with $p_{0} \in U$ there is a $q \in U$ with $q \notin S$. Let $C(X, G)=\mathscr{D} \oplus \mathscr{D}_{0}$. Then $G=G\left(p_{0}, \mathscr{D}\right)$ $\oplus G\left(p_{0}, \mathscr{D}_{0}\right)$. We claim one of $G\left(p_{0}, \mathscr{D}\right), G\left(p_{0}, \mathscr{D}_{0}\right)$ has local direct factors and hence $G$ does too.

Let $F \subseteq G, F$ finite. Let $\pi_{1}, \pi_{2}$ be the projections of $G$ onto $G\left(p_{0}, \mathscr{D}\right)$ and $G\left(p_{0}, \mathscr{D}_{0}\right)$ respectively. Say $\pi_{1}(F)=\left\{g_{1}, \ldots, g_{k}\right\}$ and $\pi_{2}(F)=\left\{h_{1}, \ldots, h_{t}\right\}$. Let $f_{i} \in \mathscr{D}, 1 \leqslant i \leqslant k$, and $f_{j}^{\prime} \in \mathscr{D}_{0}, 1 \leqslant j \leqslant t$, be such that $f_{i}\left(p_{0}\right)=g_{i}$ and $f_{j}^{\prime}\left(p_{0}\right)=h_{j}$. Let $U_{i}=f_{i}^{-1}\left(g_{i}\right), V_{j}=f_{j}^{\prime-1}\left(h_{j}\right), 1 \leqslant i<k, 1 \leqslant j \leqslant t$. Now set $V=\cap_{1<i<k} U_{i} \cap$ $\cap_{1<j<t} V_{j} . V$ is open and $p_{0} \in V$. Thus there is a $q \in V, q \notin S$. We have $\left\{g_{1}, \ldots, g_{k}\right\} \subseteq G(q, \mathscr{D})$ while $\left\{h_{1}, \ldots, h_{t}\right\} \subseteq G\left(q, \mathscr{D}_{0}\right)$. Further $G(q, \mathscr{D}) \neq$ $G\left(p_{0}, \mathscr{D}\right)$ and $G\left(q, \mathscr{D}_{0}\right) \neq G\left(p_{0}, \mathscr{D}_{0}\right)$.

Case 1. $G\left(p_{0}, \mathscr{D}\right) \nless G(q, \mathscr{D})$. Then $\pi_{1}(F) \subseteq G\left(p_{0}, \mathscr{D}\right) \cap G(q, \mathscr{D})<G\left(p_{0}, \mathscr{D}\right)$.

Case 2. $G\left(p_{0}, \mathscr{D}\right)<G(q, \mathscr{D})$. Then since $\Delta(G)$ is Boolean, $G\left(q, \mathscr{D}_{0}\right)<G\left(p_{0}, \mathscr{D}_{0}\right)$ and $\pi_{2}(F) \subseteq G\left(p_{0}, \mathscr{D}_{0}\right) \cap G\left(q, \mathscr{D}_{0}\right)=G\left(q, \mathscr{D}_{0}\right)<G\left(p_{0}, \mathscr{D}_{0}\right)$.

We can assign the finite subsets of $G$ to $Y_{1}, Y_{2}$ as follows: $F \in Y_{1}$ if $\pi_{1}(F)$ is in a proper factor of $G\left(p_{0}, \mathscr{D}\right) ; F \in Y_{2}$ if $\pi_{2}(F)$ is in a proper factor of $G\left(p_{0}, \mathscr{D}_{0}\right)$. One of $Y_{1}$ or $Y_{2}$ must be cofinal in the finite subsets of $G$. If it is $Y_{1}$ then $G\left(p_{0}, \mathscr{D}\right)$ has local direct factors, if $Y_{2}, G\left(p_{0}, \mathscr{D}_{0}\right)$ does.

When $\operatorname{Hom}(G, \zeta G)=0, C(X, \Delta(G))$ is a Boolean algebra and we can define a Boolean monomorphism $\Phi: C(X, \Delta(G)) \rightarrow \Delta(X, G)$ as follows: any $\varphi \in$ $C(X, \Delta(G))$ has a unique representation as $\varphi\left(U_{1}, D_{1}\right) \vee \cdots \vee \varphi\left(U_{n}, D_{n}\right)$ where $\varphi\left(U_{i}, D_{i}\right)$ is defined in a manner analogous to $f\left(U_{i}, g_{i}\right)$ and where $\left\{U_{i} \mid 1<i<n\right\}$ partitions $X$ while $D_{1}, \ldots, D_{n}$ are distinct elements of $\Delta(G)$. Let $\Phi(\varphi)=\delta\left(U_{1}, D_{1}\right)$ $\oplus \cdots \oplus \delta\left(U_{n}, D_{n}\right)$. It is routine to check that $\Phi$ is a Boolean monomorphism. Notice that if $\Phi$ is onto $\Delta(X, G)$, we have the isomorphism $\Delta(X, G)=\Delta(C(X, G))$ $\cong C(X, \Delta(G))$ of Boolean algebras. This roughly says that the Boolean power functor commutes with the direct factor functor.

Theorem 2. Assume $\operatorname{Hom}(G, \zeta G)=0$ and $\mathscr{D} \in \Delta(X, G)$. Then $\mathscr{D} \in \operatorname{range}(\Phi)$ if and only if $G(, \mathscr{D})$ is continuous. Hence $\Phi$ is onto $\Delta(X, G)$ if and only if $G(, \mathscr{D})$ is continuous for all $\mathscr{D} \in \Delta(X, G)$.

Proof. Suppose $\mathscr{D} \in \Delta(X, G) \cap \operatorname{range}(\Phi)$ and let $\varphi \in C(X, \Delta(G))$ with $\Phi(\varphi)=$ Q . Let $\varphi\left(U_{1}, D_{1}\right) \vee \cdots \vee \varphi\left(U_{n}, D_{n}\right)$ be the unique representation of $\varphi$. Then $\mathscr{D}=\delta\left(U_{1}, D_{1}\right) \oplus \cdots \oplus \delta\left(U_{n}, D_{n}\right)$. For $x \in U_{j}, G(x, \mathscr{D})=D_{j}$. Thus, $G(, \mathscr{D})$ is constant on each $U_{j}$ and $G(, \mathscr{D})$ is continuous.

Conversely, suppose $\mathscr{D} \in \Delta(X, G)$ and $G(, \mathscr{D})$ is continuous. There is a partition $\left\{U_{i} \mid 1 \leqslant i \leqslant n\right\}$ of $X$ and distinct $H_{i} \in \Delta(G), 1 \leqslant i<n$, such that $G(x, \mathscr{D})=$ $H_{i}$ for $x \in U_{i}$. If $f \in \mathscr{D}, f\left(U_{i}\right) \subseteq H_{i}$. Thus $D<\delta\left(U_{1}, H_{1}\right) \oplus \cdots \oplus \delta\left(U_{n}, H_{n}\right)$. We show $\mathscr{D}=\delta\left(U_{1}, H_{1}\right) \oplus \cdots \oplus \delta\left(U_{n}, H_{n}\right)$ by showing the generators $f(V, h)$ of each $\delta\left(U_{i}, H_{i}\right)$ are in $\mathscr{D}$. Fix $f(V, h)$ with $V \subseteq U_{i}, h \in H_{i}$. For each $x \in V$ there is an $f_{x} \in \mathscr{D}$ with $f_{x}(x)=h$. Let $V_{x}=f_{x}^{-1}(h)$. $\left\{V_{x} \mid x \in V\right\}$ covers $V$. Since $X$ is 
compact and $V$ is clopen, $V=V_{x_{1}} \cup \cdots \cup V_{x_{m}}$ for some $x_{1}, \ldots, x_{m} \in V$ and we can assume $V_{x_{i}} \cap V_{x_{j}}=\varnothing$ for $i \neq j$. By the projection lemma $f\left(V_{x_{i}}, h\right) \in \mathscr{D}$, $1 \leqslant i \leqslant m$. But $f(V, h)=f\left(V_{x_{1}}, h\right)+\cdots+f\left(V_{x_{m}}, h\right)$ and $f(V, h) \in \mathscr{D}$. Clearly $\mathscr{D}=\Phi(\varphi)$ where $\varphi=\varphi\left(U_{1}, H_{1}\right) \vee \cdots \vee \varphi\left(U_{n}, H_{1}\right)$.

Corollary. If $\operatorname{Hom}(G, \zeta G)=0$ and for some Boolean algebra $B$ the mapping $\Phi$ is not onto $\Delta(X, G)$, then $G$ has local direct factors; thus if $G$ does not have local direct factors, the isomorphism $\Delta(X, G) \cong C(X, \Delta(G))$ holds for all Boolean algebras B.

This corollary is a simple consequence of Theorems 1 and 2. It applies trivially provided $G$ is indecomposable and centerless and yields $\Delta(X, G) \cong C(X, \Delta(G))=$ $C(X,\{0,1\}) \cong B$. This isomorphism was first established by Jónsson [4].

We next consider the condition of having local direct factors.

Definition. For $A \subseteq G, A^{G}$ is the normal closure of $A$ in $G$.

Theorem 3. Assume $\Delta(G)$ is Boolean and $G=K^{G}$ where $K$ is a countable set. If $G$ has local direct factors then $G$ is the direct sum of an infinite set of groups.

Proof. It is convenient to use the following fact about Boolean algebras: if $\left\langle b_{i}\right| i\langle\omega\rangle$ is an infinite sequence of elements of $B$ then there is a subsequence $\left\langle b_{i j}\right\rangle$ and another sequence $\left\langle c_{i_{j}}\right\rangle$ of elements of $B$ such that for all $i_{j}, 0 \neq c_{i j}<b_{i}$ and if $j \neq k, c_{i_{j}} \wedge c_{i_{k}}=0$. Now let $K=\bigcup\left\{K_{i} \mid i<\omega\right\}$ where $K_{i}$ is finite for each $i<\omega$. Since $G$ has local direct factors, let $G=A_{i} \oplus B_{i}$ where $K_{i}<A_{i}$ and $B_{i} \neq\{0\}$ for each $i<\omega .\left\{B_{i} \mid i<\omega\right\}$ is evidently an infinite subset of $\Delta(G)$. By our opening remarks there is a subsequence $\left\langle B_{i_{j}}\right\rangle$ and a sequence $\left\langle C_{i_{j}}\right\rangle$ of elements from $\Delta(G)$ such that for all $j, 0 \neq C_{i_{j}} \leqslant B_{i_{j}}$ and thus $C=\Sigma^{\oplus} C_{i}$, exists in $G$. We show $C \in \Delta(G)$. We proceed by constructing the complement of $C$. Let $x \in G$. Say $x \in K_{n}^{G}$. Hence for all $m>n, \pi_{C_{i_{m}}}(x)=0$. Put $x=x_{1}+x_{2}$ where $x_{1} \in C_{i_{0}}$ $\oplus \cdots \oplus C_{i_{n}}$ and $x_{2} \in \overline{C_{i_{0}} \oplus \cdots \oplus C_{i_{n}}}$ (complement). We have $\pi_{C_{i}}\left(x_{2}\right)=0$ for all $j$. Thus $x_{2} \in \cap\left\{\bar{C}_{i j} \mid j<\omega\right\}$. Let $H$ be the group generated by $\left\{x_{2} \mid x \in G\right\}$. Then $G=C \oplus H$.

Corollary. If $\operatorname{Hom}(G, \zeta G)=0, G=K^{G}$ where $K$ is countable and $G$ is not a direct sum of an infinite set of groups, then $\Phi$ is onto $\Delta(X, G)$ for all $B$ and $\Delta(X, G) \cong C(X, \Delta(G))$.

For example, if $G$ is finitely generated and $\operatorname{Hom}(G, \zeta G)=0$ then $\Delta(X, G) \simeq$ $C(X, \Delta(G))$ holds for all $B$.

If $G$ has local direct factors, we have no general criterion (involving conditions on $G$ and $B)$ to determine when $\Phi$ is onto $\Delta(X, G)$ and hence when $\Delta(X, G) \simeq$ $C(X, \Delta(G))$ holds. However, we can give a complete answer when $G$ is an infinite direct sum of groups.

Theorem 4. Let $B$ be an infinite Boolean algebra and suppose $\operatorname{Hom}(G, \zeta G)=0$. If $G$ is a direct sum of an infinite set of groups, then $\Phi$ is not onto $\Delta(X, G)$. 
Proof. Let $G=\sum_{n=1}^{\infty}{ }^{\oplus} G_{i}$. By Theorem 2 it suffices to produce a $\mathscr{D} \in \Delta(X, G)$ for which $G(, \mathscr{D})$ is not continuous. It is well known that in any infinite Boolean algebra there exists a countable set of nonzero elements whose pairwise intersections are zero. Let $\left\{U_{i} \mid 1 \leqslant i<\omega\right\}$ be such a collection in $X$, the Stone space of $B$.

We describe $\mathscr{D}$ and $\mathscr{D}_{0}$ by telling which generators of $C(X, G)$ are in them. For $V \subseteq X$,

(i) if $V \cap U_{n}=\varnothing$ for all $n$ then $f(V, g) \in \mathscr{D}$ for all $g \in G$,

(ii) if $V \cap U_{m} \neq \varnothing$ and $V \cap U_{m} \neq V$ while $V \cap U_{j}=\varnothing$ for all $j<m$, then $f(V, g) \in \mathscr{D}$ for all $g \in G_{1}+\cdots+G_{m}$,

(iii) if $V \cap U_{m}=V$ then $f(V, g) \in \mathscr{D}$ for all $g \in G_{1}+\cdots+G_{m}$ and $f(V, g) \in$ $\mathscr{D}_{0}$ for all $g \in \sum_{m+1<i} G_{i}$.

$\mathscr{D}$ and $\mathscr{D}_{0}$ are generated by the above designated elements.

We claim $C(X, G)=\mathscr{D} \oplus \mathscr{D}_{0}$. It suffices to show that each $f\left(U, g_{k}\right) \in \mathscr{D}+\mathscr{D}_{0}$ where $g_{k} \in G_{k}, 1 \leqslant k<\omega$, and that $\mathscr{D} \cap \mathscr{D}_{0}=\{0\}$.

Case 1. $U \cap U_{n}=\varnothing$ for all $n$. From (i) we have $f\left(U, g_{k}\right) \in \mathscr{D}$.

Case 2. $U \cap U_{n}=U$ for some $n$. We apply (iii). If $n>k, f\left(U, g_{k}\right) \in \mathscr{D}$ and if $k>n, f\left(U, g_{k}\right) \in \mathscr{D}_{0}$.

Case 3. $l$ is the first index for which $U \cap U_{l} \neq \varnothing$ or $U$ while $U \cap U_{j}=\varnothing$ for all $j<l$ and $l \geqslant k$. Then from (ii) we have $f\left(U, g_{k}\right) \in \mathscr{D}$.

Case 4. Same conditions as Case 3 except $l<k$. From (iii) $f\left(U \cap U_{l}, g_{k}\right) \in \mathscr{D}_{0}$. Consider $V=U \cap \bar{U}_{l}$. By our choice of $l$, if $V \cap U_{j} \neq \varnothing$ then $j>l$. We want to show $f\left(V, g_{k}\right) \in \mathscr{D}+\mathscr{D}_{0}$. This would allow us to conclude $f\left(U, g_{k}\right)=$ $f\left(U \cap U_{l}, g_{k}\right)+f\left(U \cap \bar{U}_{l}, g_{k}\right) \in \mathscr{D}+\mathscr{D}_{0}$. Either Case 1, 2, or 3 allows us to say $f\left(V, g_{k}\right) \in \mathscr{Q}+\mathscr{D}_{0}$ or we are in the circumstances of Case 4. That is, $m$ is the least index for which $V \cap U_{m} \neq \varnothing$ or $V$ and $V \cap U_{j}=\varnothing$ for all $j<m$ and $m<k$. Note of course $m>l$. As above we conclude $f\left(V \cap U_{m}, g_{k}\right) \in \mathscr{D}_{0}$ and we consider $f\left(V \cap \bar{U}_{m}, g_{k}\right)$. Again either Case 1, 2, or 3 apply or we are in Case 4 for $V \cap \bar{U}_{m}$. As the index of the first intersecting $U_{j}$ is rising, this process terminates in a finite number of steps. We then see that $f\left(V, g_{k}\right) \in \mathscr{D}+\mathscr{D}_{0}$ and thus $f\left(U, g_{k}\right) \in \mathscr{D}+$ $\mathscr{D}_{0}$. Hence $C(X, G)=\mathscr{D}+\mathscr{D}_{0}$.

Now we show $\mathscr{D} \cap \mathscr{D}_{0}=\{0\}$. Let $h \in \mathscr{D} . h=f\left(V_{1}, g_{1}\right)+\cdots+f\left(V_{n}, g_{n}\right)$ where $f\left(V_{i}, g_{i}\right)$ is a generator of $\mathscr{D}$ as described in (i), (ii), or (iii). In the case of types (ii) and (iii) the relevant index $m$ is considered part of the type. It is easy to see that generators of distinct types commute and that generators of like types can be combined. Thus we may assume $h=f\left(V_{1}, g_{1}\right)+\cdots+f\left(V_{n}, g_{n}\right)$ where $f\left(V_{i}, g_{i}\right)$ is a generator of $\mathscr{D}$ and $V_{1}, \ldots, V_{n}$ are pairwise disjoint. The same remarks apply to $h \in \mathscr{D}_{0}$. If $h \in \mathscr{D} \cap \mathscr{D}_{0}$ and $x \in X$, by our above comments there are generators $f\left(V_{1}, g_{1}\right)$ and $f\left(V_{0}, g_{0}\right)$ of $\mathscr{D}$ and $\mathscr{D}_{0}$ respectively such that $h(x)=f\left(V_{0}, g_{0}\right)(x)$ $=f\left(V_{1}, g_{1}\right)(x)$ and $x \in V_{0} \cap V_{1}$. From the definition of $\mathscr{D}_{0}, V_{0} \subseteq U_{m}$ for some $m$ and $g_{0} \in \Sigma_{m+1 \leqslant i} G_{i}$. Now $x \in V_{0} \cap V_{1}$ and we have $V_{1} \cap U_{m} \neq \varnothing$. Hence $f\left(V_{1}, g_{1}\right)$ is either of type (ii) or (iii). In either case $g \in \Sigma_{i<j} G_{i}$ where $j<m$. But $\sum_{i<m} G_{i} \cap \sum_{m+1<i} G_{i}=\{0\}$ and hence $g_{0}=g_{1}=0$. Thus $h \equiv 0$ and $\mathscr{D} \cap \mathscr{D}_{0}=$ $\{0\}$. 
We complete the proof by showing $G(, \mathscr{D})$ is not continuous. Let $S=$ $\cup_{1<i<\omega} U_{i}, T=\bar{S}=\bigcap_{1<i<\omega} \bar{U}_{i} . T$ is closed and nonempty. $T$ is not open. If it were, $\left\{U_{i}\right\}, T$ would be an open cover of the compact space $X$ which has no finite subcover. Thus there is a $p \in T, p \notin$ interior of $T$. So each neighborhood of $p$ contains points of $S$. Let $N_{0}$ be a neighborhood of $p$. There is a least $m_{0}$ such that $U_{m_{0}} \cap N_{0} \neq \varnothing$. Let $N_{1}=N_{0} \cap \bar{U}_{m_{0}} \cdot p \in N_{1}$ so there is a least $m_{1}$ with $U_{m_{1}} \cap N_{1}$ $\neq \varnothing$. Note $m_{0}<m_{1}$. Continuing this process, we get a sequence of integers $m_{0}<m_{1}<\ldots$, and a sequence of neighborhoods of $p, N_{0} \supset N_{1} \supset \ldots$ such that $U_{m_{i}} \cap N_{i} \neq \varnothing$. From the definition of $\mathscr{D}$ it is easy to see that $G(p, \mathscr{D})=\sum_{i=1}^{\infty}{ }^{\oplus} G_{i}$ $=G$. Let $N$ be any neighborhood of $p$ and let $m$ be the least integer with $U_{m} \cap N \neq \varnothing$. Choose any $q \in U_{m} \cap N$. Since $q \in U_{m}, G(q, \mathscr{D})=G_{1}$ $+\cdots+G_{m}$. Thus $G(, \mathscr{Q})$ is not continuous at $p$.

Next we give an example of a group $G$ possessing "countably local direct factors" but which is not isomorphic to an infinite direct sum of nontrivial groups. This $G$ is a group for which Theorems 1 and 2 are of no use in analyzing $\Delta(X, G)$. For such a group we do not know if there must exist a Boolean algebra $B$ such that $\Delta(X, G)$ has "discontinuous" members (not in the range of $\Phi$ ).

The construction of $G$ is an easy direct limit of Boolean powers. Let $B$ be a countable atomless Boolean algebra and let $I \subsetneq B$ be a maximal ideal. Let $H$ be a nonabelian simple group and let $P=C(X, H)$ be the Boolean power of $H$ with respect to $B$. Let $M$ be the subgroup of $P$ generated by $\{f(U, g) \mid U \in I, g \in H\}$. $M$ is the direct sum of infinitely many subgroups $P_{D} \cong P$ where every factor $P_{D}$ corresponds to an element $D$ of a fixed pairwise disjoint generating set of $I$ and $P_{D}=\langle f(U, g) \mid U \leqslant D, g \in H\rangle=\delta(D, H)$.

We define a chain of groups under subgroup inclusion $\left\{G_{\alpha} \mid \alpha<\omega_{1}\right\}$ as follows. Put $G_{0}=P$. The crucial properties of the construction will be

(a) For all $\alpha<\omega_{1}, G_{\alpha} \cong P$.

(b) For all $\alpha<\beta<\omega_{1}, G_{\alpha}$ is a direct factor of $G_{\beta}$.

Suppose $G_{\alpha} \cong P$ has been defined. Let $\varphi: G_{\alpha} \rightarrow M$ embed $G_{\alpha}$ as a direct factor $P_{D}$ of $M$ and let $G_{\alpha} \subset G_{\alpha+1}$ be isomorphic to the embedding $G_{\alpha} \stackrel{\varphi}{\rightarrow} M \subset P$. This embeds $G_{\alpha}$ as a direct factor of $G_{\alpha+1}$ since every direct factor $P_{D}$ of $M$ is also a direct factor of $P$. Now suppose $\alpha$ is a limit ordinal and $G_{\beta}$ is defined for all $\beta<\alpha$ and (a) and (b) are satisfied. Using (b) we decompose $A=\cup\left\{G_{\beta} \mid \beta<\alpha\right\}$ into an infinite direct sum of subgroups $A_{i}(0 \leqslant i<\omega)$ with every $A_{i}$ isomorphic to a direct factor of some $G_{\beta} \cong P$; specifically let $\left\langle\beta_{i}\right| i\langle\omega\rangle$ be cofinal in $\alpha$ and let $A_{i}$ be such that $G_{\beta_{i+1}}=A_{i} \oplus G_{\beta_{i}}$. Thus every $A_{i} \cong P$ (all nontrivial direct factors of $P$ are isomorphic to $P$ ) and $A \cong M$. We may choose $G_{\alpha}$ so that the extension $G_{\alpha} \supset A$ is isomorphic to $P \supset M$. Since every $G_{\beta}(\beta<\alpha)$ is a direct factor of some $G_{\beta_{i}}, G_{\beta}$ is a direct factor of $G_{\alpha}$ also.

Now let $G=\bigcup\left\{G_{\alpha} \mid \alpha<\omega_{1}\right\}$. Using (b) we see that for all $\alpha<\omega_{1}, G_{\alpha} \in \Delta(G)$. Hence $G$ has "countably local direct factors": every countable subgroup of $G$ is contained in a proper direct factor of $G$. We claim $G$ cannot be the direct sum of 
infinitely many nontrivial subgroups. To obtain a contradiction suppose $G=$ $\sum_{i=1}^{\infty} \oplus_{i}\left(L_{i} \neq 0\right)$. Choose $\alpha<\omega_{1}$ so that $G_{\alpha} \cap L_{i} \neq 0$ for all $i . G_{\alpha} \cong P$ is the normal closure of a single element (since $H$ is nonabelian and simple) contrary to $G_{\alpha}$ meeting all the direct factors of $L_{i}$ of $G$.

2. Isomorphism types. Let $P(G, B)$ be the isomorphism type of the bounded Boolean power of $G$ with respect to $B$. Lawrence calls a group $G$ Boolean separating ( $B$-separating) if $B \mapsto P(G, B)$ is one-to-one on all isomorphism types $B$. In [4] it was shown that every nontrivial, indecomposable, centerless $G$ (actually $\operatorname{Hom}(G, \zeta G)=0$ is sufficient) is $B$-separating in the strong sense that $B$ can be recovered from $P(G, B)$ as $\Delta(P(G, B)) \cong B$.

Before presenting our first theorem we need the

Definition. $\zeta_{G}(H)=$ the centralizer of the subgroup $H \subseteq G$ in $G$. A nonabelian group $G$ is centrally indecomposable if and only if $G$ cannot be expressed as a sum $G=H+K$ of nonabelian subgroups $H$ and $K$ with $K \subseteq \zeta_{G}(H)$. Notice that if $G=H+K$ as above, then $H \cap K \subseteq \zeta G$ and $G \cong H+K / \Omega$ where $\Omega \subseteq \zeta H+$ $\zeta K$ and $\Omega \cap \zeta H=\Omega \cap \zeta K=0$.

THEOREM 5. If $G$ is nonabelian and centrally indecomposable, then $G$ is B-separating.

Notice that every centerless, indecomposable group is centrally indecomposable.

Lawrence [6] has shown that:

(1) No abelian group is $B$-separating;

(2) No direct square $G \times G$ is $B$-separating (since there are Boolean algebras $B_{0} \cong B_{1}$ with $B_{0} \times B_{0} \cong B_{1} \times B_{1}$, and $C(X, G \times G) \cong C(X \times X, G)$ is easily checked); and

(3) Every nonabelian, finite, subdirectly irreducible group is $B$-separating.

We enlarge Lawrence's positive result to

TheOReм 6. Let $G$ be a nonabelian group in which every two nontrivial normal subgroups intersect nontrivially. $G$ is $B$-separating.

Our final result is motivated by proofs occurring in Shelah [7], Baldwin and Saxl [1], and Belegradek [2] that every countable nonabelian group has $2^{\kappa}$ subcartesian powers in every cardinal $\kappa>\aleph_{0}$. In fact for $\kappa>\aleph_{0}$ and regular there are $2^{\kappa}$ mutually nonembeddable such powers. These proofs all use logical instability which says nothing in the countable case $\kappa=\kappa_{0}$. Boolean powers do settle the problem in this case, at least for finitely generated groups.

THEOREM 7. Every nonabelian finitely generated group $G$ has $2^{\kappa_{0}}$ nonisomorphic countable Boolean powers.

Of course Boolean powers cannot be expected to provide mutually nonembeddable groups as in stability theory where delicate set theory is used.

Theorems 5 and 6 considerably increase the class of directly indecomposable groups known to be $B$-separating from [4] and [6]. Let $\chi$ and $\Lambda$ be the classes of nonabelian groups of Theorems 5 and 6 , repectively. Thus members of $\Lambda$ satisfy 
the "normal filter" property of Theorem 6 while members of $\chi$ are centrally indecomposable. These classes are somewhat independent because of the central product construction for groups: if $G \in \Lambda$ and $\zeta G \neq 0$ then every central power $P$ of $G$ with amalgamated center $\left(P=\Sigma^{\oplus}\left\{G_{\alpha} \mid \alpha \in I\right\} / \Omega\right.$ where $G_{\alpha} \cong G$ and $\Omega=\left\langle z_{\alpha}\right.$ $\left.-z_{\beta}|\alpha \neq \beta \in I, z \in \zeta G\rangle\right)$ also belongs to $\Lambda$ because, for $\alpha \in I, \zeta P=\zeta G_{\alpha}$ since the centers of the $G_{\alpha}$ are amalgamated in $P$, and every nontrivial normal subgroup of $P$ intersects some $G_{\alpha} \subseteq P$ nontrivially. This gives many members of $\Lambda-\chi$. For example, let $F(n)$ be the free class 2 nilpotent group on $n$ generators. Let $F(2)=$ $\langle a, b\rangle . \zeta F(2)$ is infinite cyclic with generator $[a, b]$ and every nontrivial normal subgroup of $F(2)$ meets $\zeta F(2)$ nontrivially. Thus $F(2) \in \Lambda$, so every central power of $F(2)$ with amalgamated center is in $\Lambda$ too (but not in $\chi$, except for $F(2)$ itself). Notice that if $n>2$, then $F(n) \notin \Lambda$ because $\zeta F(n)$ has $\operatorname{rank}(n(n-1) / 2)$. But it is not difficult to show that $F(n) \in \chi$ (free nilpotent groups of any nilpotency class should be in $\chi$ also). An indecomposable group which none of our theorems show to be $B$-separating is $F(n) \times F(n) / \Omega(n>2)$ with $\Omega=\{(z,-z) \mid z \in \zeta F(n)\}$ (amalgamated center). We do not know if this type of group is $B$-separating. So, in spite of our positive results, the problem of characterizing $B$-separating indecomposable groups remains quite open. We have no example of a nonabelian indecomposable group which is not $B$-separating. Another class of interest is $\Psi=\{G \mid \operatorname{Hom}(G, \zeta G)=0\}$. We know from $\S 1$ that indecomposable members of $\Psi$ are $B$-separating since $\Delta(X, G) \cong B$ (Jónsson's proof in [4] extends to this case as well). This result is evidently not contained in Theorems 5 and 6, again because of central amalgamations: if $G \in \Psi$, then every central power $P$ of $G$ with amalgamated centers is in $\Psi$ also and $P$ will be indecomposable provided $G$ is centrally indecomposable.

In the proof of Theorem 7 we select a certain class of $2^{\boldsymbol{k}_{0}}$ nonisomorphic, countable, atomic Boolean algebras and we show that every nonabelian finitely generated group $G$ "separates" all the members of this class. The special structure of these Boolean algebras provides means for recovering $B$ from $C(X, G)$ not available when dealing with arbitrary Boolean algebras as in Theorems 5 and 6. In fact the proof of Theorem 7 reduces to two cases: either $G$ is nilpotent or $G$ is centerless. In the first case we show that if $B$ is atomic, then a fixed finite cartesian power $B^{m}$ of $B$ can be isomorphically recovered from any Boolean power $C(X, G)$ of $G$ over $B$, thus guaranteeing $B$-separation for atomic $B$ except in cases similar to Lawrence's example- $B_{1} \approx B_{2}$ but $B_{1}^{m} \cong B_{2}^{m}$-which are the only nonabelian counterexamples to $B$-separation we know of. In the case that $G$ is centerless we do not do quite as well-we recover the normal subgroup series of $C(X, G)$ corresponding to the Tarski ideal series of $B$. (The Tarski ideal of $B$ is generated by all atoms and atomless elements of $B$.) The clarification of this situation will likely be facilitated by Ketonen's recent classification theorem for countable Boolean algebras which we do not attempt here.

Proof of THEOREM 5. First we make the

Definition. Let $B$ be a Boolean algebra with Stone space $X$ and let $G$ be a group. Let $\mathscr{D} \in \Delta(X, G)$. 
(a) $G(x, \mathscr{D})=\mathscr{D}(x)=\{f(x) \mid f \in \mathscr{D}\}$ (this is the evaluation map notation of $\S 1)$.

(b) $\gamma(\mathscr{D})=\{x \in X \mid G(x, \mathscr{D}) \Varangle \zeta G\}$.

(c) $\chi$ is the class of nonabelian centrally indecomposable groups.

Lemma 2. Suppose $G \in \chi, C(X, G)=\mathscr{D}_{0} \oplus \mathscr{D}_{1}$, and $x \in X$. Then there is $a$ unique $i=0$ or 1 such that $G\left(x, \mathscr{D}_{i}\right) \nless \zeta G\left(i . e ., x \in \gamma\left(\mathscr{D}_{i}\right)\right)$ and for this value of $i$ we have $\zeta_{G}\left(G\left(x, \mathscr{D}_{i}\right)\right)=\zeta G$.

Proof. Since $C(X, G)=\mathscr{D}_{0} \oplus \mathscr{D}_{1}$, it is easy to see that $G=G\left(x, \mathscr{D}_{0}\right)+$ $G\left(x, \mathscr{D}_{1}\right)$ where the subgroups $G\left(x, \mathscr{D}_{i}\right)$ commute elementwise (i.e., $G$ is a central product of these subgroups) and since $G \in \chi$, exactly one of these subgroups is nonabelian and the other is contained in $\zeta G$. It remains to verify the final equality. We have $\zeta G=\zeta G\left(x, \mathscr{D}_{0}\right)+\zeta G\left(x, \mathscr{D}_{1}\right)=\zeta_{G}\left(G\left(x, \mathscr{D}_{i}\right)\right)$ because $G\left(x, \mathscr{D}_{1-i}\right) \subseteq \zeta G$.

Lemma 3. Let $\mathscr{D} \in \Delta(X, G)$. Then $\gamma(\mathscr{D})$ is clopen.

Proof. Put $C(X, G)=\mathscr{D} \oplus \mathcal{E}$. Choose $a \in G-\zeta G$ and put $f(X, a)=d+e$ where $d \in \mathscr{D}, e \in \mathcal{E}$. We claim that for all $x \in X$

$$
x \in \gamma(\mathscr{D}) \text { (i.e., } G(x, \mathscr{D}) \Varangle \zeta G) \text { if and only if } d(x) \notin \zeta G \text {. }
$$

Assuming (*) the proof that $\gamma(\mathscr{D})$ is clopen is to put $d=f\left(U_{1}, a_{1}\right)$ $+\cdots+f\left(U_{n}, a_{n}\right)$ where $U_{i}, 1 \leqslant i \leqslant n$, are pairwise disjoint. Then $\gamma(\mathscr{D})$ must be the union of some of the $U_{i}$.

Now for (*): the reverse implication is immediate since $d(x) \in \mathscr{D}(x)=G(x, \mathscr{D})$. To prove the forward implication contrapositively assume $x \in X$ and $d(x) \in \zeta G$. Then $e(x) \notin \zeta G$ and by Lemma $2, x \in \gamma(\mathcal{E})-\gamma(\mathscr{D})$ (because exactly one of $G(x, \mathscr{D})$ and $G(x, \mathcal{E})$ is central in $G)$.

LEMMA 4. Let $C=C(X, G)$. If $\mathcal{E}, \mathscr{D} \in \Delta(X, G)$, then $\zeta_{C}(\mathcal{E}) \subseteq \zeta_{C}(\mathscr{D})$ if and only if $\gamma(\mathscr{D}) \subseteq \gamma(\mathcal{E})$.

Proof. $(\Leftarrow)$ Suppose $\gamma(\mathscr{D}) \subseteq \gamma(\mathcal{E})$ and $f \in \zeta_{C}(\mathcal{E})$. By Lemma $2, f(x) \in \zeta G$ for all $x \in \gamma(\mathcal{E})$ since $\zeta_{G}(G(x, \mathcal{E}))=\zeta G$. Hence $f(x) \in \zeta G$ for all $x \in \gamma(\mathscr{D}) \subseteq \gamma(\mathcal{E})$ which implies $f \in \zeta_{r}(\mathscr{D})$.

( $\Rightarrow$ Suppose $\zeta_{C}(\mathcal{E}) \subseteq \zeta_{C}(\mathscr{D})$ but $U=\gamma(\mathscr{D})-\gamma(\mathcal{E}) \neq \varnothing . \quad U$ is clopen by Lemma 3. Choose $a \in G-\zeta G$. We have $f(U, a) \in \zeta_{C}(\mathcal{E})$ since $U \cap \gamma(\mathcal{E})=\varnothing$ while $f(U, a) \notin \zeta_{C}(\mathscr{D})$ because if $x \in U \subseteq \gamma(\mathscr{D})$, then Lemma 2 says $\zeta_{G}(G(x, \mathscr{D}))$ $=\zeta G$ and hence $a \notin \zeta G$ does not centralize $G(x, \mathscr{D})$ (which implies $f(U, a) \notin$ $\left.\zeta_{C}(\mathscr{D})\right)$. So, $f(U, a) \in \zeta_{C}(\mathcal{E})-\zeta_{C}(\mathscr{D})$ which is impossible.

Completion of THE Proof of Theorem 5. By Lemma 4 , the condition $\zeta_{C}(\mathcal{E}) \subseteq$ $\zeta_{C}(\mathscr{D})$ defines a reflexive and transitive preorder $\mathscr{D} \leqslant \mathcal{E}$ on $\Delta(X, G)$. Let $<$ be the induced partial ordering of the equivalence classes $[\mathscr{D}]$ of $\Delta(X, G)$ under $\preccurlyeq$. By Lemma $4,[\mathscr{D}]=[\mathcal{E}]$ if and only if $\gamma(\mathscr{D})=\gamma(\mathcal{E})$ because $[\mathscr{D}]<[\mathcal{E}]$ if and only if 
$\gamma(\mathcal{E}) \subseteq \gamma(\mathscr{D})$. By Lemma 3, $\{\gamma(\mathscr{D}) \mid \mathscr{D} \in \Delta(X, G)\}=\{U \mid U \subseteq X\}$. Hence, $<$ defines on $[\Delta(X, G)]$ a lattice ordering isomorphic to that of $B$.

Proof of Theorem 6. If $\zeta G=0$ then $G$ is $B$-separating by Jónsson's Theorem [4]. So assume $\zeta G \neq 0$ and every two nontrivial normal subgroups of $G$ intersect nontrivially. Thus $\zeta G$ is a rank 1 abelian group because every subgroup of $\zeta G$ is normal in $G$. There are two cases. Either $\zeta G$ is cyclic or a quasi-cyclic $p$-group (Case 1), or else $\zeta G$ is torsion free and a subgroup of the additive rationals (Case 2). Let $B$ be a Boolean algebra, $X$ its Stone space. Let $C=C(X, G)$ be as in $\S 1$.

Case 1 . In this case $G$ is subdirectly irreducible with minimum normal subgroup $Z_{p}$ of order $p$. If $g \in C$, let $L_{g}$ consist of all the elements of

$$
[g, C]=\langle g+f-g-f \mid f \in C\rangle^{C}
$$

(normal closure in $C$ ) which have order $p$ and lie in $\zeta C$. Let $S_{g}=\{x \in X \mid g(x) \notin$ $\zeta G\}$. Noting that $\zeta C=C(X, \zeta G)$, it is easily seen that $L_{g}$ is generated by all the $f\left(V, Z_{p}\right)=\left\{f(V, a) \mid a \in Z_{p}\right\} \subseteq C$ where $V \subseteq S_{g}$ is a clopen set on which $g$ is constant. ( $f\left(V, Z_{p}\right)$ is the minimum normal subgroup of $f(V, G)$.) Hence, for all $g, h \in C, L_{g}=L_{h}$ if and only if $S_{g}=S_{h}$. Since every $V \subseteq X$ is an $S_{g}$ for some $g \in C$, the subgroups $\left\{L_{g} \mid g \in C\right\}$ form a lattice isomorphic to $B$ and $G$ is $B$-separating.

Case 2. Let us first note (*) if $N \neq 0$ is a subgroup of $\zeta G$ then $\zeta G / N$ is periodic. If $g \in C$, let $L_{g}=[g, C] \cap \zeta C$ and let $S_{g}=\{x \in X \mid g(x) \notin \zeta G\}$ as in Case 1 . Define a relation $\sim$ on $C$ as follows: if $g, h \in C$ then $g \sim h$ if and only if both $L_{g} L_{h} / L_{g}$ and $L_{g} L_{h} / L_{h}$ are periodic.

Claim. If $g, h \in C$ then $g \sim h$ iff $S_{g}=S_{h}$.

Proof of Claim. Suppose $S_{g} \neq S_{h}$. Without loss of generality assume we have $\varnothing \neq V \subseteq S_{g}-S_{h}$. Thus $[g, C] \supseteq[g, \delta(V, G)] \cap f(V, \zeta G) \neq 0$ and since $V \cap S_{h}$ $=\varnothing,[h, C] \cap f(V, \zeta G)=0$. Hence $L_{g} L_{h} / L_{h} \cong L_{g} / L_{g} \cap L_{h}$ is not periodic since it contains a nontrivial subgroup of $f(V, \zeta G)$ which is torsion free. So $h \nsim g$. On the other hand, suppose $S_{g}=S_{h}=S$. So $L_{g}, L_{h} \subseteq \delta(S, \zeta G)$ and both $L_{g}$ and $L_{h}$ intersect every $f(V, \zeta G)$ nontrivially where $V \subseteq S$. In view of (*) this implies both $\delta(S, \zeta G) / L_{g}$ and $\delta(S, \zeta G) / L_{h}$ are periodic. Hence $h \sim g$ and the claim is established.

Thus $\sim$ is an equivalence relation on $C$. We partially order the equivalence classes $C / \sim$ as follows: $[g] \leqslant[h]$ if and only if $L_{g} L_{h} / L_{h}$ is periodic. It is easy to check, as in the claim, that $[g] \leqslant[h]$ if and only if $S_{g} \subseteq S_{h}$. Since every clopen set is $S_{g}$ for some $g \in C$, the ordering $<$ on $C / \sim$ is isomorphic to (the Boolean lattice) $B$ and, since $\sim$ and $\leqslant$ are definable in $C$ we conclude that $G$ is $B$-separating.

Proof of Theorem 7. The proof is divided into two cases according to whether $G$ equals the largest term $\zeta^{\infty} G$ of its transfinite upper central series $\cdots \zeta^{\alpha} G<$ $\zeta^{\alpha+1} G \cdots$ defined by $\zeta^{0} G=0, \zeta^{\alpha+1} G / \zeta^{\alpha} G=\zeta\left(G / \zeta^{\alpha} G\right)$ for ordinals $\alpha$ with continuity at limit ordinals. If $G=\zeta^{\infty} G$, then since $G$ is finitely generated, simple commutator calculus shows the last limit group $\zeta^{\lambda} G$ must itself be finitely normally generated in $G$. Hence $G$ is nilpotent. This will be Case 2. 
Case 1. $G \neq \zeta^{\infty} G$. Put $F=G / \zeta^{\infty} G$. It is routine to check by induction that, for all ordinals $\alpha$ and Boolean algebras $B, \zeta^{\alpha} C(X, G)=C\left(X, \zeta^{\alpha} G\right)$. (This would be true in any "bounded" subcartesian power of $G$ in which all functions have finite range in $G$.) Hence $H=\zeta^{\infty} C(X, G)=C\left(X, \zeta^{\infty} G\right)$ and we can recover from $C(X, G)$ the group

$$
K=C(X, G) / H \cong C(X, F) .
$$

Now $F \neq 0$ is centerless, so $\Delta(K)$ is Boolean. By Theorems 1,2 , and 3 the canonical mapping $\Phi: C(X, \Delta(F)) \rightarrow \Delta(K)$ defined as in $\S 1$ by $\varphi(U, D) \mapsto \delta(U, D)$ where $U \subseteq X, D \in \Delta(F)$ is onto $\Delta(K)$.

Claim. Suppose $0 \neq \mathscr{D} \in \Delta(K)$. Then

(1) $\mathscr{D}$ is finitely generated if and only if $\mathscr{D}=\delta\left(\left\{p_{1}\right\}, D_{1}\right) \oplus \cdots \oplus \delta\left(\left\{p_{n}\right\}, D_{n}\right)$ for some isolated points $p_{1}, \ldots, p_{n} \in X$ and $D_{i} \in \Delta(F)$.

(2) $\mathscr{D}$ has no finitely generated direct factors if and only if $\mathscr{D}=\delta\left(U_{1}, D_{1}\right)$ $\oplus \cdots \oplus \delta\left(U_{n}, D_{n}\right)$ where each $U_{i}, 1 \leqslant i \leqslant n$, has no isolated points and $D_{i} \in$ $\Delta(F)$.

Proof of Claim. Since $F$ is finitely generated, every $\delta(\{p\}, D) \simeq D$ is finitely generated where $p$ is an isolated point of $X$ and $D \in \Delta(F)$. On the other hand if $U \neq \varnothing$ is not just a finite set of isolated points and $0 \neq D \in \Delta(F), \delta(U, D)$ is not finitely generated since every finite subset of $\delta(U, D)$ lies in some subgroup $f\left(U_{1}, D\right) \oplus \cdots \oplus f\left(U_{n}, D\right) \neq \delta(U, D)$ where $\left\{U_{i} \mid 1<i<n\right\}$ is a partition of $U$. Both parts of the claim easily follow from these observations.

Define $R=R(B)$ to be the ideal of $B$ generated by all the atoms of $B$ together with the atomless elements of $B$. Inductively put $R_{1}=R$ and $R_{n+1} / R_{n}=$ $R\left(B / R_{n}\right)$.

For $b \in B$, let $U_{b} \subseteq X$ be the clopen set corresponding to $b$. If $I$ is an ideal of $B$, define $I(X, F) \subseteq C(X, F)=K$ to be the join of the subgroups $\left\{\delta\left(U_{b}, F\right) \mid b \in I\right\}$. It is well known that $K / I(X, F) \cong C(X-I, F)$ where $X-I$ is the Stone space of $B / I$.

By the previous claim and the above definitions we have

(i) $R(X, F)$ is the join of all $\mathscr{D} \in \Delta(K)$ where $\mathscr{D}$ is either finitely generated or $\mathscr{D}$ has no nontrivial finitely generated direct factors.

Having found $R_{n}(X, F)$, we have

(ii) $R_{n+1}(X, F) / R_{n}(X, F)$ is the join of $\mathscr{D} \in \Delta\left(K / R_{n}(X, F)\right)$ where $\mathscr{D}$ has the properties enunciated in (i). Consequently the subgroup series $\left\{R_{n}(X, F) \mid n>1\right\}$ can be defined in $K$. Also

(iii) $B / R_{n}$ has an atomless element if and only if $K / R_{n}(X, F)$ has a direct factor with no finitely generated subfactors.

It is well known that there are $2^{\kappa_{0}}$ countable atomic Boolean algebras which differ in the sets of values of $n$ for which $B / R_{n}(B)$ has atomless elements. In the Stone space this means: in the sequence $X=X^{0} \supseteq X^{1} \supseteq X^{2} \supseteq \ldots$ where $X^{n+1}$ $=$ limit points of $X^{n}$, there are values of $n$ for which $X^{n}$ contains points which are not the limits of isolated points. Constructing such Boolean algebras involves combinatorially the partitioning of an infinite set of atoms obtained at stage $n$ into infinitely many new (stage $n+1)$ atoms and optionally a "dense" portion as well. 
These values of $n$ can be recovered from $P(B, F)=$ isomorphism type of $C(X, F)$ in accordance with (i)-(iii) giving $2^{\kappa_{0}}$ nonisomorphic Boolean powers of $F$ and hence of $G$.

Case 2. $G$ is nilpotent. Let $\mathcal{Q}$ be the class of $2^{\kappa_{0}}$ countable atomic Boolean algebras used in the preceding case. We will show that $G$ separates $\mathbb{Q}$, i.e., the map $B \rightarrow P(G, B)$ is one-to-one on isomorphism types for $B \in \mathbb{Q}$. A simple fact about $Q$ we will use later is (0) if $B_{0} \approx B_{1} \in \mathbb{Q}$ and $m \geqslant 1$, then $B_{0}^{m} \approx B_{1}^{m}$ (since a direct product does not affect the stages at which atomless elements occur in the $R$-series).

Assume $B \in \mathbb{Q}$ and let $C=C(X, G)$ where $X$ is the Stone space of $B$. For $a \in G$ define $[a, G]$ to be the normal closure of the set of commutators $\{a+g-$ $a-g \mid g \in G\}$. Let $\pi_{x}$ be the projection of $C$ at $x \in X$; so $\pi_{x}(f)=f(x)$. Put $\operatorname{supp}(f)=\{x \in X \mid f(x) \neq 0\}$.

We use the following easily checked facts.

(1) For all $f \in C$ and $x \in X, \pi_{x}([f, C])=[f, C](x) \neq 0$ if and only if $f(x) \notin \zeta G$.

(2) Let $f, h \in C$. If $f(x)=h(x)$ for all $x \in \operatorname{supp}(f)$ then $[f, C] \subseteq[h, C]$; indeed, if $h=f\left(U_{1}, g_{1}\right)+\cdots+f\left(U_{n}, g_{n}\right) \quad\left(U_{i}\right.$ 's pairwise disjoint) then $[h, C]=$ $\Sigma^{\oplus}\left[f\left(U_{i}, g_{i}\right), C\right]$.

(3) If $G$ is any group, $g \in G$, and $z \in \zeta G$ then $[g, G]=[g+z, G]$.

Put $r=$ the minimum general rank among the abelian subgroups $\{[f, C] \cap \zeta C \mid f$ $\in C-\zeta C\}$. (All of the abelian subgroups in this set are nontrivial because every nontrivial normal subgroup of the nilpotent group $C$ meets $\zeta C$ nontrivially.) Hence $r>1$. Suppose $f \in C$ with $\operatorname{supp}(f)=\{x\}$ where $x$ is an isolated point of $X$. Then by (1), $[f, C] \cap \zeta C \subseteq \zeta \delta(\{x\}, G) \cong \zeta G$ which is finitely generated since $G$ satisfies the maximum condition on its subgroups. Thus $1 \leqslant r$ is finite.

Put $S=\{h \in C \mid[h, C] \cap \zeta C$ has rank $r\}$. Notice that $S=S+\zeta C$ by (3). We claim

(4) For all $h \in S$ there is a unique isolated point $x \in X$ with $h(x) \notin \zeta G$.

If two such points $x_{1}, x_{2}$ existed, then $[h, C] \cap \zeta C$ would have, according to (2), direct factors of rank $\geqslant r$ at both $x_{1}$ and $x_{2}$ coordinates and thus would have rank $\geqslant 2 r$, contrary to $h \in S$. On the other hand, some such $x$ must exist because $B \in \mathbb{Q}$ is atomic (the isolated points of $X$ are dense).

Define an equivalence relation $\sim$ on the cosets $S / \zeta C$ as follows:

If $H=\left\{h_{1}+\zeta C, \ldots, h_{n}+\zeta C\right\}$ is a finite subset of $S / \zeta C$, then $H$ is called a related set provided there exists an additive dependence relation among the subgroups $\left\{\left[h_{i}, C\right] \cap \zeta C \mid 1 \leqslant i \leqslant n\right\}$, i.e., there exist $z_{i} \in\left[h_{i}, C\right]$ (not all $z_{i}=0$ ) such that $\sum z_{i}=0$. Let $R=\{H \subseteq S / \zeta C \mid H$ is a minimal related set $\}$ and let $\sim$ be the equivalence relation on $S / \zeta C$ generated by the subsets $R$, i.e., every $H \in R$ is a subset of a single $\sim$ equivalence class.

For each isolated point $x \in X$ put $S_{x}=\{h \in S \mid h(x) \notin \zeta G\}$. By (4) there is exactly one such $x$ for every $h \in S$. Note also that $h+\zeta C \subseteq S_{x}$ for all $h \in S_{x}$. We claim

(5) If $H \in R$, then for some isolated point $x \in X, H \subseteq S_{x}$; hence every $\sim$-equivalence class of $S / \zeta C$ is contained in some $S_{x} / \zeta C$; and 
(6) For every $x \in X$, every $n+1$-element subset of $S_{x} / \zeta C$ is related, where $n=\operatorname{rank}(\zeta G)$. Hence, for every isolated point $x \in X, S_{x} / \zeta C$ is divided into $m \leqslant n$ equivalence classes under $\sim(m$ is the same for all $x)$.

Proof of (5). If a related set $H$ contained members of both $S_{x}$ and $S_{y}$ with $x \neq y$ isolated points of $X$, then $H$ would not be minimal since [ $\left.H \cap S_{x}, C\right]$ and $\left[H \cap S_{y}, C\right]$ are independent.

Proof of (6). The first part is obvious. That $m$ is fixed is clear since $m$ depends only on the properties of $G$ and not on $x$.

We next show that a Boolean algebra isomorphic to $B^{m}$ is generated by a definable collection $\left\{T_{g} \mid g \in C\right\}$ of subsets of $S / \zeta C$. If $f \in S$, let $[f]$ denote the $\sim$-equivalence class of $f+\zeta C$. We define $T_{g}$ for $g \in C$ by $T_{g}=\{[f] \mid f \in S$ and $[g, C] \cap[f, C] \cap \zeta C \neq 0\}$. Let $x \in X$ be a fixed isolated point, let $\varphi \in S_{x}$ and $U \subseteq X$. Put $T(U, \varphi)=\left\{\left[f_{y}\right] \mid y \in U, y\right.$ isolated, $f_{y} \in S_{y}$, and $\left.f_{y}(y)=\varphi(x)\right\}$. Thus $T(U, \varphi)$ picks out the $\sim$-equivalence classes $\left[f_{y}\right] \subseteq S_{y}$ (for all the isolated $y \in U$ ) which correspond to the class $[\varphi] \subseteq S_{x}$ under the coordinate-indexing of copies of $G$. Notice that $x$ simply fixes a coordinate copy of $G$ for reference. We claim

(7) Under set theoretic operations, the sets $\left\{T(U, \varphi) \mid \varphi \in S_{x}, U \subseteq X\right\}$ generate a Boolean algebra $\mathcal{T}_{0}$ isomorphic to $B^{m}$; and, letting $\mathcal{T}$ be the Boolean algebra generated by the sets $\left\{T_{g} \mid g \in C\right\}$, we have

(8) $\mathcal{T} \subseteq \mathcal{T}_{0}$ and

(9) $\mathcal{T}_{0} \subseteq \mathcal{T}_{\text {. }}$

The proof of (7) is completely routine using the fact that $B \in \mathbb{Q}$ is atomic.

Proof of (8). Every $T_{g} \in \mathcal{T}$ is seen to be the union of finitely many sets of the form $T(U, \varphi)$ by considering the unique representation of $g$ as $\sum f\left(U_{i}, g_{i}\right)=\sum f_{i}$ since $[g, C]=\Sigma\left[f_{i}, C\right]$ and $T_{g}=\cup\left\{T_{f_{i}}\right\}$ and every $T_{f_{i}}$ is a certain finite union of the form $T_{f_{i}}=T\left(U_{i}, \varphi_{1}\right) \cup \cdots \cup T\left(U_{i}, \varphi_{k}\right)$ where $\varphi_{j} \in S_{x}$ correspond to the $\sim$ equivalence classes which happen to nontrivially intersect $\left[f_{i}(y), G\right] \cap \zeta G(y \in$ $\left.U_{i}\right)$.

Proof of (9). Let $\varphi \in S_{x}$ and $U \subseteq X$. We claim

$$
T(U, \varphi)=T_{f(U, \varphi(x))}
$$

Clearly $\subseteq$ holds. So, equality will follow (consulting the definition of $T_{g}$ and recalling (4)) provided: if $f \in S_{y}$ for some isolated $y \in U$ and if $[f(y), G] \cap$ $[\varphi(x), G] \cap \zeta G \neq 0$ then $[f]=\left[\varphi_{y}\right]$ where $\varphi_{y} \in S_{y}, \varphi_{y}(y)=\varphi(x)$. Now $[f(y), G] \cap$ $[\varphi(x), G] \cap \zeta G \neq 0$ implies $\left\{f, \varphi_{y}\right\}$ is a minimal related set whence $[f]=\left[\varphi_{y}\right]$ follows.

Thus $B^{m}$ is definable in $C(X, G)$ and (in view of (0)) the proof is complete.

\section{REFERENCES}

1. J. T. Baldwin and J. Saxl, Logical stability in group theory, J. Austral. Math. Soc. Ser. A 21 (1976), 267-276.

2. O. V. Belegradek, On unstable theories of groups, Izv. Vysš. Učebn. Zaved. Matematika 22 (8) (1978), 41-44. 
3. J. M. G. Fell and A. Tarski, On algebras whose factor algebras are Boolean, Pacific J. Math. 2 (1952), 297-318.

4. B. Jonsson, On isomorphism types of groups and other algebraic systems, Math. Scand. 5 (1957), 224-229.

5. B. Jónsson and A. Tarski, Direct decompositions of finite algebraic systems, Notre Dame Math. Lectures, no. 5, Notre Dame, Ind., 1947.

6. J. Lawrence, $A$ note on Boolean powers of groups (preprint).

7. S. Shelah, Why there are many nonisomorphic models for unsuperstable theories, Proc. Internat. Congr. Math., Vancouver, 1974, pp. 259-263.

Department of Mathematics, Michigan State University, East Lansing, Michigan 48824 